\title{
ENERGY EFFICIENT GRID SPLITTING RELAY NODE SELECTION IN VANETS
}

\author{
Er. Ankita Dhiman ${ }^{1} \&$ Er.Amarpreet Singh ${ }^{2}$
}

Abstract-VANET network is created by range of vehicles in which vehicles communicates to the other vehicles by transferring the data packets from one node to a different or to a new. The transmission process should be effective but it can get afflicted if the nodes are located at the far distance from one another. The huge distance between nodes causes an increment in the jump counts due to which the energy of the network gets exhausted previous work. Therefore, it is necessary to enhance the network efficiency by decreasing the amount of hop counts with the minimum end to end delay. This paper provides a novel approach which is a power effective grid system for VANETs. It divides the network into the sub section which brings about a lowering in the space from one node to another node. The results of the job are also depicted in the result section. This is an evidence study that ensures that the recommended technique can work better and can be considered for further enhancements.

Keywords-VANETs, Routing, Energy, Hop Counts, Packet Delivery ratio, End-to-End Delay.

\section{INTRODUCTION}

VANETs are vehicular ad hoc networks are specifically used to guarantee the road safety by facilitating the communication among road side units such as cars, scooters and vehicles, etc. This communication can be done only within a specific range such as $100 \mathrm{~m}$ to $300 \mathrm{~m}$. Due to its features the VANETs has become an important part of the Intelligent Transport system [2]. Since VANET is developed for human safetyand comfort, it has several applications. The safety applications include collision warning, collecting traffic information, blind crossing prevention, work zone warning, weather information, etc. Its comfort applications comprise parking lot payment, internet service such as sending emails, downloading files, etc. The main purpose of both the applications is toprovide accurate information to the driver and the users of the car. This life critical information should beprecise and securely transmitted from the source to the destination [4].For transmitting the data between small ranges of communication in order to obtain the inter-vehicular communication (IVC), the vehicles are required to be installed with the communication devices. Moreover, the surplus of newly developed services that includes vehicle positioned near to each other, like navigation security, games that can be played online, and exchange of information [1]. The scenario of traffic on the road is shown through the gateways or internet gateways that can be determined at irregular time periods along with the path that can give large bandwidth connection for a short range. Due to rapid motion, the cars are in the range of each internet gateway for a short duration of time.

\subsection{Open research challenges and possible solutions for vehicular networks}

The recent main research challenges of VANETs is the requirement of centralized communication coordinator connected with all of the existing wireless access technologies utilized for VANETs implementation and deployment. VANETs are a kind of MANETs but it has some different fundamentals. Some of the research challenge regarding power control and management, routing in VANETs is defined under this section [2].In a work author has donethe analysis of both the modes i.e. normal mode and covert mode via using the four parameters like end to end delay which means that total time taken by the transmission between sender and receiver, Packet drop which calculates the number of lost packets during the transmission , Packet Delivery ratio which is the ratio of total packet received to the total packet sent during the transmission and the last parameter throughput which means that the volume of data sent during aparticular time [5].

\subsection{Power Control and Management}

Electric power management from the perspective of efficiency is not an issue in VANETs where as in other technologies such as LTE where installed power packs are being used as source of energy in the nodes suffers from this. Whereas the energy management in conditions of Tx Transmission Power is a suspect topic which must be resolved to perform the effective communication in the network. In an opaque vehicle network, the higher transmitting power can lead to interference to the current transmission with another transmission. In order to avoid this collision or interference of the

\footnotetext{
${ }^{1}$ M.Tech Scholar, Department of Computer Science and Engineering,ACET, Amritsar, I.K.G PTU, Punjab, India

2 Associate Professor and HOD, Department of Computer Science and Engineering, ACET, Amritsar, I.K.G PTU, Punjab, India
} 
communication, the compacted VANETs should use lower transmission power so that the communication should place effectively [6].

The adjustment of transmission ability also resolves the void of successful routing. Since the realignment of Tx brings about an increment in the throughput and reduction in distraction occurrence.

\subsection{Routing in VANETs}

Several researches are conducted based on suitability of Mobile ad-hoc networks routing protocol within the VANETs i.e. vehicle ad-hoc networks. On the other hand, as a result of quick division in the network of VANETs because of varying topology and high mobility of nodes in it, the protocols used in MANETs are not well suitable for vehicular network. Along with this, in mobile ad-hoc networks at any time of time the end to end online connection can be obtained easily and the nodes that lie between the source and destination node that always found in MANETs may not be attained in the vehicular networks [7].

In Vehicle ad-hoc networks the protocols used for routing has been grouped into five categories that are as follow: first is Topology based routing protocol, second is Position based direction-finding protocol, third is group based routing protocol, next is Geo-cast routing standard protocol and fifth is Voice broadcasting routing protocol. With the help of VANETs and vehicular multi-hop network, the information between various vehicles can be transmitted easily without resorting to the static architecture. Due to dynamic topology, it becomes mandatory to implement optimum protocols. Consequently, a reasonable freeway mobility model is presented and implemented to analyze the operational efficiency of AODV, DSR, FSR and TORA in the specific dynamic traffic condition centered on network simulation [10]. After simulation the results obtained has shown that the AODV has outperformed in all types of traffic conditions implemented by FSR and DSR, on the other hand the TORA is not applicable over the vehicle networks [14].

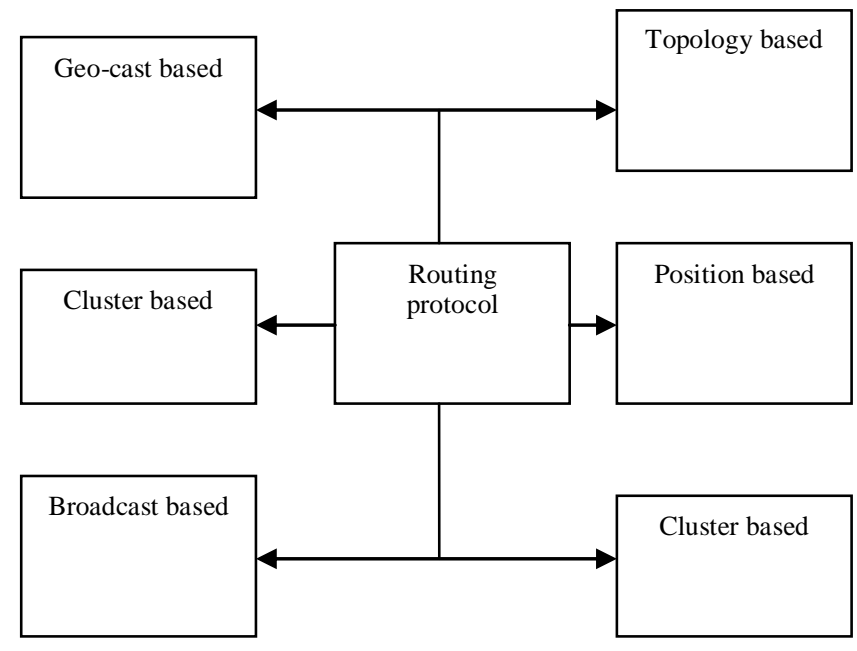

Figure 1 VANETs Routing Protocols

\subsection{Topology Based Routing Protocols}

Through this type of routing protocols, the information about the links that sustain over the network is employed to perform packet transmission. Further this protocolis split up into two parts that are: Proactive and Reactive.

\subsection{Proactive routing protocols}

In proactive routing the data is transmitted in such a way that forwarding hop is upheld in the background no subject about the transmission needs. Benefit associated with this type of protocol is the fact here it is not required to identify the route as the target route as it was accumulated in previous. Even so the demerits are also associated with this redirecting protocol and that is it provides delay in real time applications. In a node of the network the table is maintained to depict the next node in a particular hop to reach the destination node in the network. A desk is constructed and managed in a node. Along with this it also stores the unused routes for data. Different types of proactive routing protocols are: LSR, FSR

\subsection{Reactive/Ad Hoc Based Redirecting}

In this type of redirecting technique, the path will open only when the node wants to form the link with some other node in the network. In this type of routing only that paths are need to maintain that are presently being used. Therefore, in end result the overhead of network will reduces. In reactive routing the destination client is dependent upon flooding the query packets over the network and when the prospective client has reached then this phase is completed. Various kinds of reactive routing protocols are: AODV, PGB, DSR and TORA [10] 


\subsection{Position Based Routing Protocols}

The positioning based routing approach is composed of class of routing paradigm. In this paradigm the data related to the geographic position is employed to determine the next hop to reach the target node. To forward the packet in the network towards the destination node no map is necessary; the hops are selected on the most basic on closeness of client to the destination. This type of routing works well as there is no need to create a global route from source to node need. This kind of routing protocol is further classified into two types: first one is Location based greedy V2V protocols and second is Hold up Tolerant Protocols [13].

\subsection{Broadcast Routing Protocols}

In this routing protocol, the information packets are flooded within the broadcast domain in the network. The broadcast redirecting protocols are being used in vehicle ad-hoc networks. These are generally found in route breakthrough discovery technique. In numerous routing protocols like AODV, the received data packets are transmitted again in the network. Thereforein this redirecting technique, the packets reach the destination node by passing through various intermediately nodes. This also results in large amount of network bandwidth utilization by rebroadcasting the information packets. Almost all the nodes in the network should can easily eliminate the replica of information packets [14].

\subsection{Geo Cast Routing Protocols}

The Geo cast routing strategy is derived from the sot based multicast redirecting technique. The central idea of this routing standard protocol is to transmit the data packets from the origin node to various other nodes in particular region. The Geo cast redirecting protocol may be defined as multicast routing service in particular geographic area in which the specific region is defined in which the data packets are flooded to decrease the overhead and traffic jam in the network [15].

\subsection{Cluster Based Redirecting protocols}

This type of routing is implemented over the clusters. The nodes in the networks participate in the particular cluster created in the network. Every single cluster in the network composed of a cluster head and for that reason it is accountable to broadcast the packets in the cluster. Scheduled to the clusters creation in highly dynamic vehicle ad-hoc networks, the dormancy and overhead incurred in it. To make the network scalable, it is required that the redirecting virtual network should be created.

In [25] Li Xia qing, L Hui, YANG Kai and Liu Qiao has proposed a boosting range forwarding routing protocol for vehicular ad-hoc networks for city scenario and various improvements has been presented in this routing standard protocol so that it can be implemented in several cases. The improved outcomes were obtained of improved standard protocol in sparse as well as dense traffic situation. Drawback associated with it is much less secure.In [26] R. Anggoro, R. Nakamura, T. Kitasuka, T. Itokawa and M. Aritsugi has evaluated the routing protocols used in vehicular ad-hoc networks with probabilistic relay. In this paper it was described that probabilistic relay can affect the operational efficiency of OLSR and AODV. It acquired resulted in increment in mobility of vehicles and road length. The drawback associated with it was less reliability.

\subsection{Relay Node Selection}

In VANETs, data cannot be transmitted directly from one vehicle to another vehicle. In this case, a relay node is used to transmit the data. Thus, a vehicle has to select a nearer relay node to send data from one to another. A relay node may be used for VANET to gather data from the nearby transmitting nodes and forward it to the required destination node. A relay node may be a static RSU (Road Side Unit) or a mobile node. So the selection of relay node is one of the important aspects of communication in VANETs. The significance of relay node lies in terms of parameters like delay and PDR that affects the communication [9].

\section{BACKGROUND}

From the study, it has been observed that existing work deals with the concept of relay node and used different techniques, some of these techniques are furthest distance scheme, [17] nodes falling further from the source are assigned with less waiting time while contending for becoming next relay. A technique link metric, known as expected progress distance (EPD) introduced to assess transmission link quality. And fast forwarding mechanism is achieved by providing smaller waiting time to nodes residing in segments that are farther away from the source. An opportunistic and sender-oriented broadcasting protocol has been introduced to improve the speed of messages by assigning dynamic waiting time to sender-oriented relay. It measures the packets error rate for both forward and reverse links.

The BDSC bidirectional stable communication method introduced in [1] that was distributed and sender-oriented multi-hop broadcasting scheme, which was composed of three components that were responsible for the establishment/exchanges of the periodic HELLO packets, estimation of forward link qualities and adaptive selection of the available relay nodes.[17] This scheme aims to improve multi-hop packet delivery ratio over densely populated network and at far distant nodes. This relay selection scheme focuses not only on the distance from the source but also on the link quality ratio.

After having a review on the traditional work that was done in the field of VANETs to select the relay nodes, in order to increase the packet delivery ratio and reducing the distance covered by the data, it is observed that the traditional work still poses the backlogs which motivates us to develop a new approach in direction of solution to the problem. 


\section{METHODOLOGY}

The methodology that follows for the transmission in this work is as:

1. Initiate the process of transmission by designing the whole network such as number of parameters, simulation area and nodes in that area.

2. After initializing the area, design the whole network such as dividing of network into 16 blocks of $4 \times 4$.

3. Then nodes are deployed on the simulated area where each node sends its data to the other nodes using relay node.

4. The criteria behind selecting the relay node is finding through the center of each block. The node which is nearer to the center node is chosen as a relay node then other nodes send their data to that node without consuming their time in searching a relevant node for data transmission.

5. Define the node as a source and destination node which will be used in the whole simulation for the transmission of packet.

6. Commences the process of communication between nodes buy sending packets.

7. Evaluate the shortest distance route from source to the destination.

8. End simulation process.

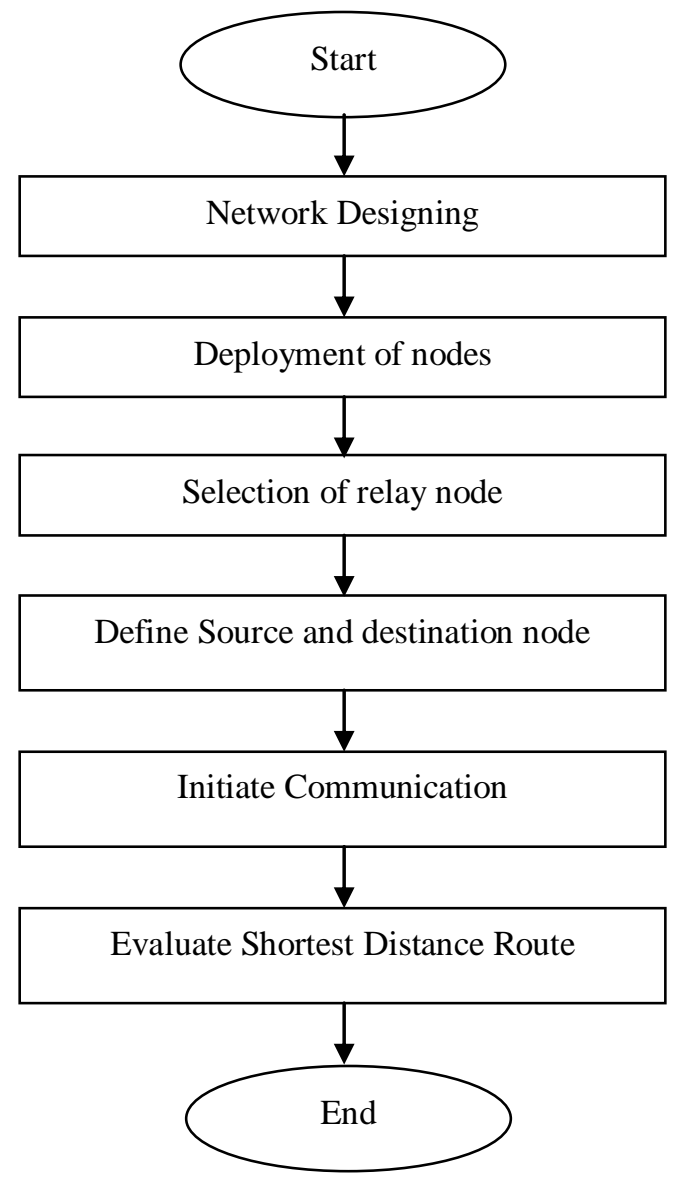

Figure 2. Framework of the proposed model

\section{IMPLEMENTATION}

In the work, the focus is to divide the whole area of the network into sub sections which will leads to the reduction in the distance covered by the data to reach the destination. The energy efficient model is also implemented to create an energy efficient grid structure. Since energy plays an important role in any sensor network asit directly effects the lifetime or the overall performance of the network. The energy model minimizes the energy consumption of non-cluster nodes by decreasing the distance between the simple nodes and nearby cluster heads. The distance is compared with the threshold value, if the distance is greater than the threshold value than the $\varepsilon_{f s}$ is used otherwise $\varepsilon_{m p g}$ energy model will be used.

In this work the relay node selection is done on the basis of distance and energy of the nodes. In each and every division of the network, there must be one relay node so that it while creating the hops for routing less distance should be covered.

On the basis of the above define work, the simulation is performed in the MATLAB and the following the graphical representations that are observed. 
The simulation parameters taken for the evaluation are shown in the below table.

Table 1. Simulation Parameters

\begin{tabular}{|l|l|}
\hline Parameter & Value \\
\hline Simulator & MATLAB \\
\hline Network studied & $\begin{array}{l}\text { Vehicular ad hoc } \\
\text { network }\end{array}$ \\
\hline Simulation area & $200 \times 200 \mathrm{~m}$ \\
\hline Nodes & $100-800$ \\
\hline Area Division & $4 \times 4$ \\
\hline Data payload & 1000 Bites / packet \\
\hline
\end{tabular}

For the simulation analysis, the parameters which are considered mentioned in the above table. In the table, simulation area is considered of $200 \times 200$ meters and nodes are varying from 100 to 800 into that area. The whole network area is divided into $4 \times 4$ blocks so in total the whole area is divided into 16 parts. The results acquired after performing simulation have shown below.

Initially, the network is designed to reduce the total time taken in selecting a relay node for the transmission of data. Considering this fact, Graphical user interface is designed where user needs to enter source and destination and then relay node has chosen in each grid. The relay node is selected based on the node which is nearer to the center node. In the above figure 3, User is requested to enter the total number of nodes in the network with the designated source as well as destination node.

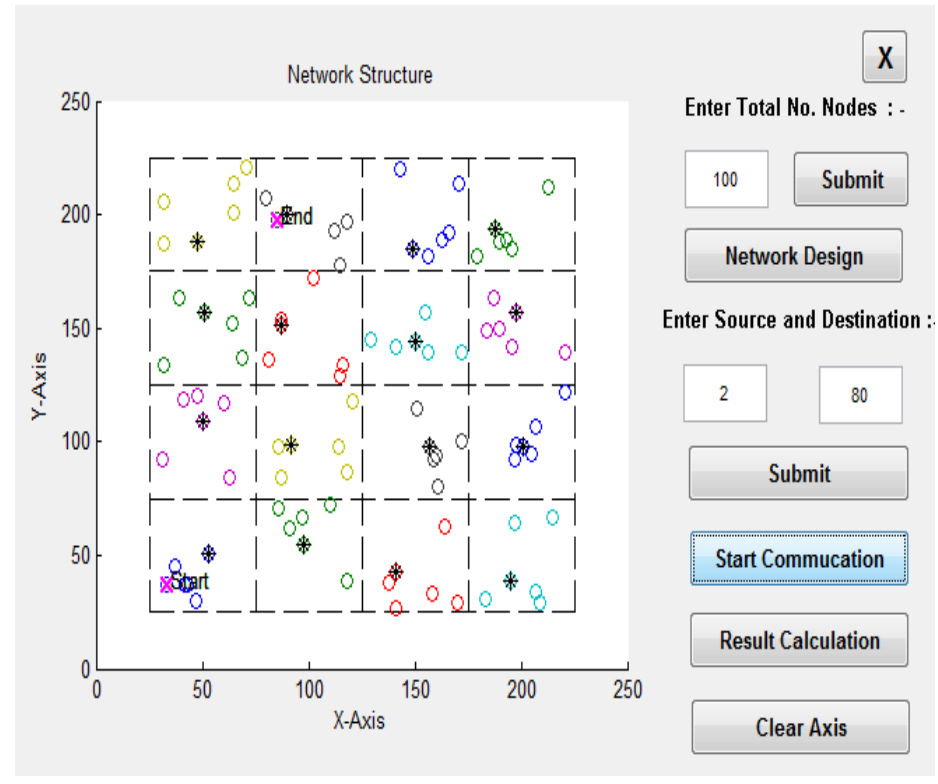

Figure 3. Network designing using EEGS technique

After initializing the network, transmission of data is performed and then performance of EEGS technique is evaluated on the basis of performance parameters such as End-to-end delay and PDR. 


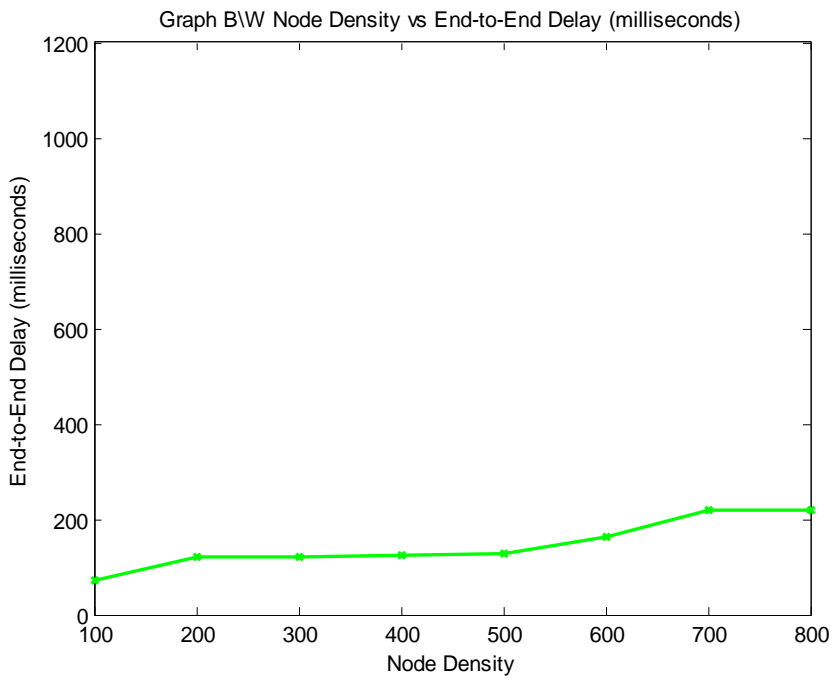

Figure 4 End-to-End Delay

The graph in figure 4 represents the end to end delay. In the graph the $\mathrm{x}$ axis shows the node density and y axis calibrates the end to end delay in milliseconds. The term end to end delay depict the time that is taken by the network to deliver the data from source node to sink node i.e. from one end to other end. From the graph it is observed that initially the end to end delay was between 50 to 70 milliseconds but as the node density gets dense or enhanced correspondingly the end to end delay also gets increased and reached nearby 220 milliseconds.

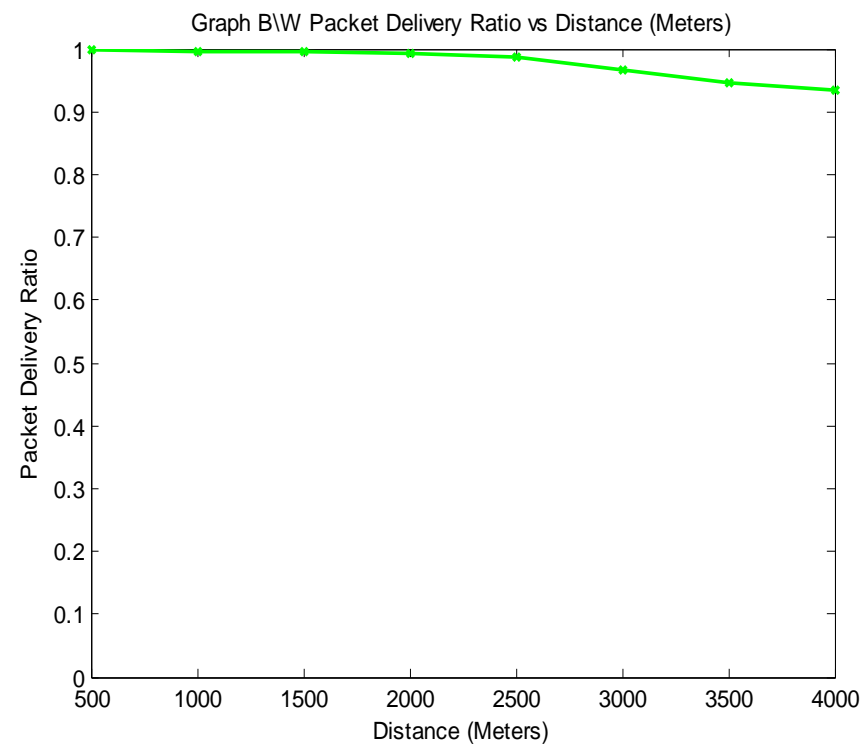

Figure 5 PDR (Packet Delivery Ratio)

The figure 5 defines the graph for packet delivery ratio. Packet Delivery Ratio performance parameter is used to estimate the total number of packets received at the destination with respect to the total number of packets sent in the network.

PDR $=\frac{\text { Pachet }_{\text {Rrgrivad }}}{\text { Packet }_{\text {Total }}}$

The graph depicts that the value of PDR is 1 initially, but it starts degrading with the enhancement in the node density. 


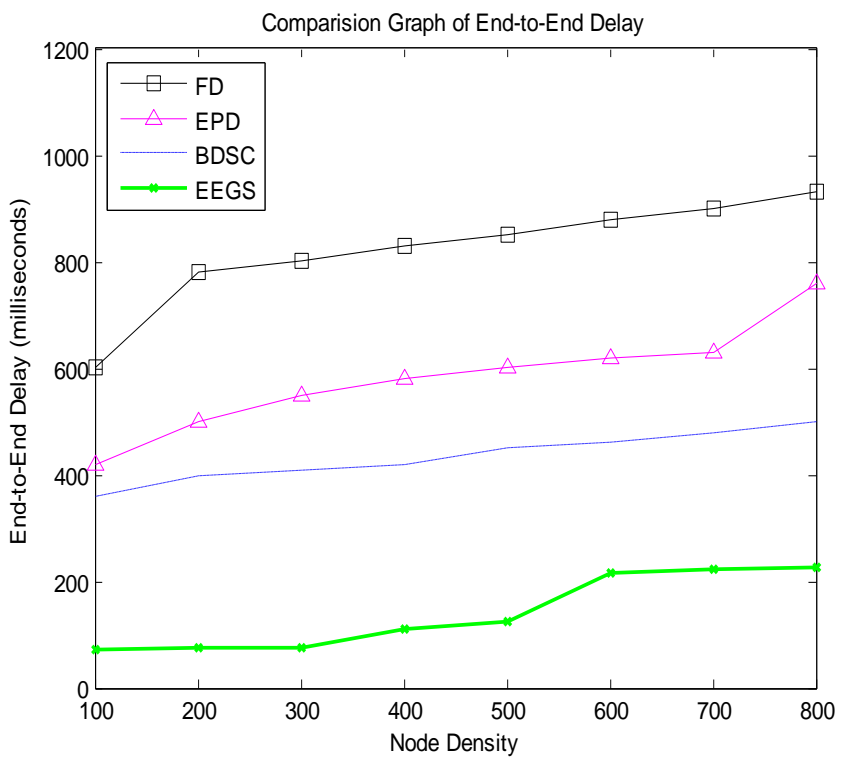

Figure 6 Comparison between different techniques in terms of End-to-End Delay

The above figure shows the comparison between different traditional techniques such as FD, EPD, BDSC and proposed EEGS. From the results acquired, it has been shown that proposed techniques takes less time from source to the destination to send the data packet in terms of different techniques. Whereas, FD performs least with highest end to end delay. An EPD and BDSC techniques averagely performs with the variation from 300 and 500 milliseconds to 700 and 400 milliseconds and with the increase density of the node, time is also gradually increased or constant.

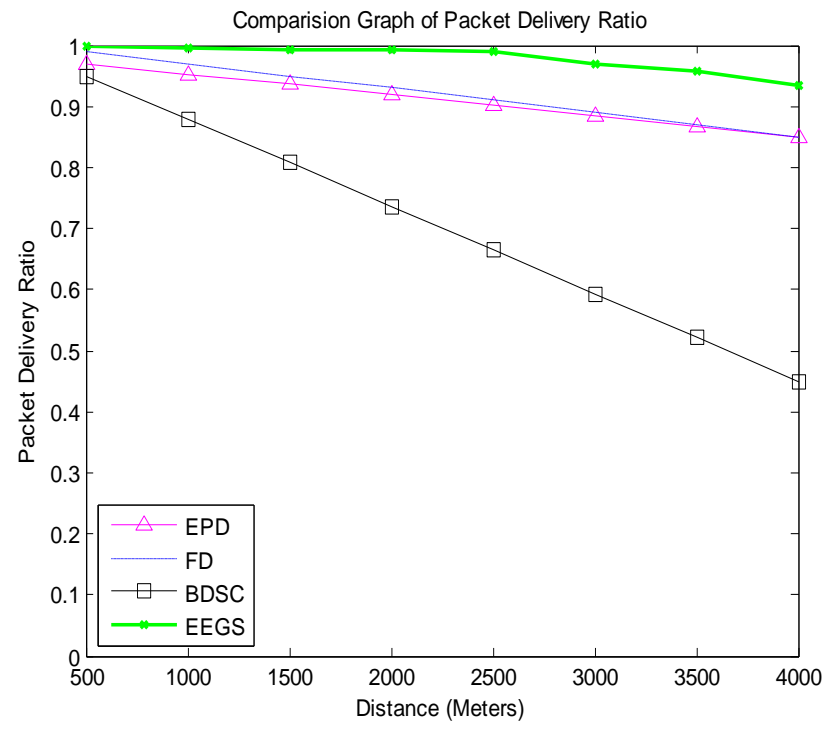

Figure 7 Comparison between different techniques in terms of Packet Delivery Ratio

The figure 7 represents the comparison between traditional and proposed technique. From the above graph, it clearly shows that proposed technique outperforms as it has maximum packet delivery ratio i.e. 1 and gradually decreased with the increase in distance. On the counter part, FD shows significant or rapid decrease in PDR with far distance.

\section{CONCLUSION AND FUTURESCOPE}

VANET is specifically deployed for providing the security system for communication among vehicles on the roads and roadside infrastructure. In this network, each and every node is mobile node thus ca move independently in the network and can communicate with any of the node in the network. Therefore the number of road accidents can be decreased by implementing the VANETs. But the node communication in the VANETs needs to improve by improving QoS parameters such as PDR, Delay etc.

This study addresses the issue of relay node selection while creating a path for data transmission. In earlier work the relay nodes were selected but these nodes were located at a quite far from each other. Due to this the data has to cover a larger distance to reach the destination and also the nodes which takes part in the routing consumes a lot of energy for transmitting 
the data at such a farther distance. In order to resolve these issues, an approach is suggested in this study which elects the relay nodes selection by using an energy model named as EEGS (Energy Effective Grid System). This study also provides a tour to the proposal technique and portrays the results in the terms of end to end delay and PDR. From the results acquired, it has shown that proposed technique outperforms the traditional technique in terms of performance parameters where it offers least delay and high packet delivery ratio.

It is concluded that in near future this approach can be implemented and a contrast can be drawn to evaluate the proficiency of the work.

\section{REFERENCES}

[1] Osama Rehman, "An adaptive relay nodes selection scheme for multi-hop broadcast in VANETs", ELSEVIER, Pp 1-15, 2016

[2] AmarpreetSingh ," Enhanced attacked packet detection algorithm for detecting dos attack in VANETS”, International Journal of Advances In Computer Science and Cloud Computing, ISSN: 2321-4058 Volume- 3, Issue- 2, Nov-2015

[3] OzanTonguz, "Broadcasting in VANETS", IEEE, 2008

[4] Swati Chaturvedi, "A Study on Broadcasting in VANETs",IJSAR, Vol 5, Issue 6, PP 2273-2276, 2016ss

[5] AmarpreetSingh ,"Evaluation of Data Transmission Using Parameters in Normal and Covert Mode in VANETS" International Journal of Advances in Electronics and Computer Science, ISSN: 2393-2835 Volume-3, Issue-1, Jan.-2016

[6] Shereen A.M. Ahmed, "A Survey on Broadcasting in VANETs", Research Journal of Applied Sciences, Engineering and Technology, Vol 7, Issue 18, PP 3733-3739, 2014

[7] Sabih-ur-Rehman, "Vehicular ad-Hoc networks (VANETs) — An overview and challenges", Computer Communications, vol. 48, pp. 141-158, 2014.

[8] O.K. Tonguz, DV-CAST: a distributed vehicular broad- cast protocol for vehicular ad hoc networks, Wireless Commun. IEEE 17 (2) (2010) $47-57$.

[9] M A. Amoroso, "Going realistic and optimal: a distributed multi-hop broadcast algorithm for vehicular safety", Comput. Netw. 55 (10) (2011) 25042519 .

[10] C. Wu , "A low latency path diversity mechanism for sender-oriented broadcast protocols in VANETs", Ad Hoc Networks 11 (7) (2013) 2059-2068

[11] Celimuge Wu, "Packet Size-Aware Broadcasting in VANETs With Fuzzy Logic and RL-Based Parameter Adaptation", IEEE, Vol 3, PP 2481-2491, 2015

[12] Celimuge Wu , "Efficient Broadcasting in VANETs Using Dynamic Backbone and Network Coding”, IEEE, Vol 14, Issue 11, PP 6057-6071, 2015

[13] Razvan Cristian Voicu , "Fast and reliable broadcasting in VANETs using SNR with ACK decoupling", IEEE, 2014

[14] AmarpreetSingh , "A Survey on Data Dissemination Protocols used in VANETs “ (International Journal of Computer Applications (0975 - 8887) Volume 120 - No.23, June 2015 Vehicular Adhoc Network (VANET)

[15] Rakesh Kumar , “A Review of various VANET data dissemination protocols”, International Journal of u-and-e services and technology, Vol 5, Issue 3, Pp 1-18, 2012

[16] Arif Sari , "Review to the security issues in VANET", International journal on communication, network and system science, vol 8, Pp 552-566, 2015

[17] Shereen A.M. Ahmed, "Survey on broadcasting in VANETs", Research journal of Applied Science, engineering and technology, Vol 7, Issue 18, Pp 3733-3739, 2014

[18] RiyanksChourse , "Review on vehicular Adhoc Network and broadcasting mechanism", International journal of scientific and engineering research, Vol 4, Issue 11, 2013

[19] Julio A. Sanguesa , "A Survey and Comparison study of broadcasting warning message dissemination schemes for VANETs", Hindawi, vol 2016, Pp $1-18,2016$

[20] Si Ho Cha , "A Survey of broadcasting protocols for VANETs", Smart computing Review, vol 4, Issue 4, Pp 1-10, 2014

[21] Sooksan Panichpapiboon, "A Review of information dissemination protocol for Vehicular Adhoc networks", IEEE, vol 14, Issue 3, 2011

[22] A. Rahim , "A Comparative Study of Mobile and Vehicular Adhoc Networks", International Journal of recent trends in engineering, vol 2, Issue 4, Pp $1-3,2009$

[23] Xiaonin Ma, "Design and analysis of robust broadcast scheme for VANET safety related services", IEEE, vol 61, Issue 1, Pp 45-61, 2011

[24] Yu Tian Tsang, "A Vehicle density based forwarding scheme for emergency message broadcast in VANETs", IEEE, 2010

[25] Luciano Bononi, “A Cross Layered MAC and clustering scheme for efficient broadcast in VANETs", IEEE, 2008

[26] Peiyuan Lai et al, "A reliable broadcast routing scheme based on mobility prediction for VANETs", IEEE, 2009

[27] SheraliZeadally et al, "Vehicular Ad hoc Network: status, results and challenges", Springer, vol 50, issue 4, Pp 217-241, 2012

[28] X. Li, "An Optimization Forwarding Range Routing Protocol for VANET in City Environments", Chinese Journal of Electronics, vol. 23, no. 1, January2014.

[29] R. Anggoro, “An evaluation of routing protocols with probabilistic relay in VANETs", TENCON 2011 - 2011 IEEE Region 10 Conf., (2011), pp. 187191 